\title{
Estudo prospectivo de avaliação de dor e incapacidade de pacientes operados de estenose de canal lombar com seguimento mínimo de dois anos
}

\author{
Prospective analysis of pain and disability of patients operated on \\ lumbar spinal stenosis with a minimum two-year follow-up \\ Estudio prospectivo de la evaluación del dolor e incapacidad de los \\ pacientes operados de estenosis del canal lumbar con seguimiento \\ mínimo de dos años
}

\author{
Edgar Santiago Valesin Filho' \\ Fabricio Hidetoshi Ueno ${ }^{2}$ \\ Luciano Temporal Borges Cabral \\ Adriano Masayuki Yonezaki² \\ Rodrigo Junqueira Nicolau² \\ Luciano Miller Reis Rodrigues ${ }^{3}$
}

\section{RESUMO}

Objetivo: realizar uma análise prospectiva de dor e incapacidade em pacientes operados de estenose de canal lombar após dois anos do procedimento através da escala VAS e Roland Morris. Métodos: trinta e oito pacientes foram avaliados por meio dos questionários em um momento pré-operatório, pós-operatório um mês, seis meses, um ano e dois anos, tendo sido realizada descompressão e artrodese com instrumentação pedicular associada. Resultados: foi observado melhora nas análises comparativas de dor e incapacidade no decorrer do seguimento em relação aos valores iniciais, porém uma tendência à estabilização do quadro com sua evolução. Conclusão: o tratamento cirúrgico da estenose do canal lombar, quando criteriosamente indicado, melhora a dor e a incapacidade após dois anos de seguimento.

\section{ABSTRACT}

Objective: to perform a prospective analysis of pain and disability in patients operated on lumbar spine stenosis two years after the procedure by means of VAS and Roland Morris scales. Methods: thirty-eight patients answered questions before and after the surgery, within one-month, sixmonth, one-year and two-year analyses; decompression and artrodesis were performed. Results: we observed a reduction in the numbers of VAS and Roland Morris scales in the comparisons with the initial values, with a tendency to stabilize with the follow-up. Conclusion: the surgical treatment of lumbar spine stenosis when correctly diagnosed (carefully indicated) improve pain and disability after two years of follow-up.
\end{abstract}

\section{RESUMEN}

Objetivo: realizar un análisis prospectivo del dolor e incapacidad en pacientes operados de estenosis del canal lumbar después de dos años del procedimiento, por medio de la escala VAS y Roland Morris. Métodos: treinta y ocho pacientes fueron evaluados por medio de los cuestionarios en un momento preoperatorio, postoperatorio un mes, seis meses, un año y dos años, realizando una descompresión y artrodesis con instrumentación pedicular asociada. Resultados: fue observada mejoría en los análisis comparativos de dolor e incapacidad con el decorrer de los seguimientos en relación a los valores iniciales; sin embargo, hubo una tendencia a la estabilidad del cuadro con su evolución. Conclusión: el tratamiento quirúrgico de la estenosis del canal lumbar, con indicación criteriosa, mejora el dolor y la incapacidad después de dos años de seguimiento.

\footnotetext{
Trabalho realizado no Hospital Estadual Mario Covas, Disciplina de Doenças do Aparelho Locomotor da Faculdade de Medicina do ABC - FMABC - Santo André (SP), Brasil.

'Estagiário de Grupo de Cirurgia da Coluna Vertebral da Faculdade de Medicina do ABC - FMABC - Santo André (SP), Brasil.

${ }^{2}$ Médico Assistente do Grupo de Cirurgia da Coluna Vertebral da Faculdade de Medicina do ABC - FMABC - Santo André (SP), Brasil.

${ }^{3}$ Mestre, Coordenador do Grupo de Cirurgia da Coluna Vertebral da Faculdade de Medicina do ABC - FMABC - Santo André (SP), Brasil. 
DESCRITORES: Medição da dor; Estenose espinhal/ cirurgia; Complicações pós-operatórias; Região lombossacral/patologia
KEYWORDS: Pain measurement;

Spinal stenosis/surgery;

Postoperative complications;

Lumbosacral region/

pathology
DESCRIPTORES: Dimensión

del dolor; Estenosis espinal/

cirugía; Complicaciones

postoperatorias; Región

lumbosacra/patología

\section{INTRODUÇÃO}

A estenose de canal vertebral foi descrita em 1954 por Verbiest como um estreitamento do canal vertebral, do recesso lateral ou dos forames neurais através da hipertrofia degenerativa progressiva de qualquer estrutura osteocartilaginosa e ligamentar circundante, podendo resultar em compressão neurológica ou vascular em um ou mais níveis ${ }^{1}$. A estenose de canal lombar é muito frequentemente uma consequência de alterações degenerativas avançadas, como hipertrofia das articulações facetárias, herniações e abaulamentos discais, hipertrofia de ligamento amarelo, formações osteofitárias, espondilolistese degenerativa e combinações entre estas condições ${ }^{2}$. A estenose do canal lombar (ECL) é uma condição dolorosa e potencialmente incapacitante comumente encontrada acometendo principalmente a população idosa, que hoje apresenta um potencial significativo de crescimento em nosso meio ${ }^{3}$. É uma causa muito frequente de dor lombar baixa e a principal indicação para cirurgia de coluna lombar em pacientes com mais de 65 anos nos Estados Uni$\operatorname{dos}^{4,5}$. A claudicação neurogênica intermitente é a principal causa de comprometimento de mobilidade e perda de independência em idosos ${ }^{6}$.

Em pacientes sintomáticos, a ECL possui quatro formas distintas de apresentação, as chamadas síndromes características: a clássica claudicação neurogênica, a dor radicular de membros inferiores, a dor lombar baixa axial e dor referida não-radicular ${ }^{7,8}$. Entretanto, a maioria destes pacientes possui sintomas que impedem uma definição exata de sua forma de apresentação, sendo na maioria das vezes a queixa principal considerada em conjunto com as demais. O diagnóstico é realizado através de história, exame físico, radiografias de coluna em incidência anteroposterior e perfil, avaliações radiográficas dinâmicas, estudos eletrofisiológicos e ressonância magnética, exame considerado de excelência para a avaliação precisa dos níveis e graus de compressão. $\mathrm{O}$ diagnóstico preciso é essencial na escolha da modalidade terapêutica ${ }^{2}$. A sensibilidade e especificidade da imagem de ressonância magnética na investigação do paciente com estenose de canal lombar é considerada superior aos outros exames de imagem como a mielografia e tomografia axial computadorizada ${ }^{7}$.

Existe uma grande quantidade de opções de tratamento conservador, demonstrando-se, através de vários estudos na literatura, uma melhora progressiva em 15 a $43 \%$ dos pacientes durante um acompanhamento de 1 a 5 anos $^{9}$. Já o tratamento cirúrgico tem como objetivo descomprimir e, de acordo com a necessidade, estabilizar a área estenosada, visando ao alívio de sintomas e à estabilização da progressão desta afecção em pacientes com alteração neurológica progressiva e à refratariedade ao tratamento clínico ou terapias minimamente invasivas.

O objetivo deste estudo foi avaliar se o procedimento cirúrgico com descompressão posterior e artrodese posterolateral da coluna lombar traz benefícios em avaliações prospectivas de dor e incapacidade.

\section{MÉTODOS}

Foram avaliados 38 pacientes com diagnóstico estabelecido de ECL por meio de exame de tomografia e ressonância magnética lombar. Inicialmente, todos foram tratados clinicamente com medicações orais analgésicas, anti-inflamatórias e fisioterapia sem sucesso por um período mínimo de seis meses. A indicação cirúrgica e os níveis de fusão foram estabelecidos de acordo com achados clínicos, em concordância com os exames de imagem, tomografia e ressonância magnética lombar e avaliações neurofisiológicas (ENMG). O objetivo da cirurgia foi alívio da dor e melhora da incapacidade funcional.

A cirurgia foi realizada através de um acesso posterior com hemilaminectomia ou laminectomia, dependendo do caso, para realizarmos a descompressão do canal vertebral medular e liberação dos forames vertebrais. Foi associado artrodese posterolateral com enxerto autólogo da crista ilíaca e instrumentação pedicular.

Os pacientes foram autorizados a deambular no terceiro dias após o procedimento cirúrgico; não foi utilizado qualquer tipo de imobilização; solicitou-se uma limitação relativa às atividades diárias durante três meses, período em que as terapias de reabilitação foram utilizadas de maneira mais intensiva.

Foram aplicados questionários de incapacidade e dor no período pré-operatório e durante o seguimento pósoperatório de um mês, seis meses, um ano e anualmente consecutivamente.

Para avaliação de dor, foi utilizada a escala analógica VAS com variação de 0 a 10 em intensidade ${ }^{10}$. Empregouse o questionário de incapacidade Rolland Morris ${ }^{11}$, que relaciona incapacidade física e dor na coluna.

Os questionários foram aplicados por uma ortopedista especializada em cirurgia da coluna vertebral integrante do grupo de coluna da Faculdade de Medicina do ABC, que também conduziu as avaliações clínicas pré e pós-operatórias.

Adotamos o nível de significância de $5 \%(0,050)$ para a aplicação dos testes estatísticos, ou seja, quando o valor da significância calculada (p) foi menor do que 5\% $(0,050)$, observou-se uma diferença ou relação 'estatisticamente significativa'; e quando o valor da significância calculada (p) foi igual ou maior do que $5 \%(0,050)$, identificou-se uma 
TABELA 1 - Descrição de valores em análise VAS em função do tempo

\begin{tabular}{lccccccc}
\hline Bloco de variáveis & $\mathbf{n}$ & Média & Desvio padrão & Mínimo & Máximo & Mediana & Significância (p) \\
\hline VAS_pré & 38 & 8,28 & 2,09 & 3,00 & 10,00 & 9,50 & \\
VAS_1m & 38 & 4,80 & 2,70 & 0,00 & 10,00 & 5,00 & $<0,001$ \\
VAS_6m & 38 & 3,99 & 2,71 & 0,00 & 10,00 & 4,00 & \\
VAS_1a & 38 & 3,58 & 2,60 & 0,00 & 9,00 & 3,00 & \\
VAS_2a & 38 & 3,43 & 2,52 & 0,00 & 9,00 & 3,00 & \\
\hline
\end{tabular}

TABELA 2 - Descrição de valores em análise Roland Morris (RM) em função do tempo

\begin{tabular}{lccccccc}
\hline Bloco de variáveis & $\mathbf{n}$ & Média & Desvio padrão & Mínimo & Máximo & Mediana & Significância (p) \\
\hline RM_pré & 38 & 14,71 & 6,69 & 1,00 & 23,00 & 17,00 \\
RM_1m & 38 & 14,87 & 4,78 & 3,00 & 21,00 & 15,00 & $<0,001$ \\
RM_6m & 38 & 10,74 & 5,46 & 3,00 & 21,00 & 10,00 & \\
RM_1a & 38 & 9,84 & 5,00 & 1,00 & 19,00 & 9,00 & \\
RM_2a & 38 & 8,29 & 4,46 & 0,00 & 18,00 & 8,00 \\
\hline
\end{tabular}

diferença ou relação dita 'estatisticamente não-significativa'. Foi utilizado o programa SPSS (Statistical Package for Social Sciences), em sua versão 13.0, para a obtenção dos resultados.

\section{RESULTADOS}

A avaliação pré-operatória incluiu 38 pacientes, 16 homens $(42,10 \%)$ e 22 mulheres $(57,89 \%)$. A média de idade foi 66,55 anos, variação de 45 a 85 anos. A quantidade de níveis de fusão variou de 1 a 5 níveis, com média de 2,47 níveis artrodesados por paciente.

Para descrição e comparação entre os momentos de observação, foi empregado o Teste de Friedman com o intuito de verificar possíveis diferenças entre os momentos pré-operatório, um mês, seis meses, um ano, dois anos após a cirurgia, considerados variáveis longitudinais em avaliações comparativas concomitantes, em relação à dor e incapacidade (Tabelas 1 e 2). Em ambos os medidores de dor e incapacidade, observaram-se diferenças estatisticamente significativas.

Foi aplicado o Teste dos Postos Sinalizados de Wilcoxon, de modo complementar, para identificação de quais momentos de observação diferem-se dos demais, conforme demonstrado na Tabela 3.

A análise da dor através da escala VAS demonstrou valor 8,26 em média de intensidade de dor pré-operatória. Após um mês, houve alteração estatisticamente significativa em relação aos valores inicias, com média de 4,78 pontos. Na análise prospectiva consecutiva, houve queda progressiva nos valores médios observados, com média após dois anos de realização do procedimento de 3,48 pontos; diferença significativa foi observada entre as análises estatísticas comparativas entre os diferentes momentos de avaliação de dor; entretanto, as análises comparativas não apresentaram diferença estatisticamente significativa quando realizadas entre momentos pareados de observação após seis meses, conforme demonstrado na Figura 1.

A análise de incapacidade demonstrou diferença significativa quando avaliados os momentos de observação
TABELA 3 - Análise de dor (VAS) e incapacidade (Roland Morris) em momentos pareados

\begin{tabular}{lcc}
\hline \multirow{2}{*}{ Par de momentos } & \multicolumn{2}{c}{ Variável } \\
\cline { 2 - 3 } & VAS & RM \\
\hline $1 \mathrm{~m}-$ pré & $<0,001$ & 0,837 \\
$6 \mathrm{~m}-$ pré & $<0,001$ & 0,006 \\
$1^{a}-$ pré & $<0,001$ & 0,003 \\
$2^{a}-$ pré & $<0,001$ & $<0,001$ \\
$6 m-1 \mathrm{~m}$ & 0,041 & $<0,001$ \\
$1^{a}-1 \mathrm{~m}$ & 0,011 & $<0,001$ \\
$2^{a}-1 \mathrm{~m}$ & 0,011 & $<0,001$ \\
$1^{a}-6 m$ & 0,071 & 0,103 \\
$2^{a}-6 m$ & 0,085 & 0,001 \\
$2^{a}-1^{a}$ & 0,455 & 0,001 \\
\hline
\end{tabular}

individualmente em comparação aos demais (Tabela 1); entretanto, quando avaliadas de forma pareada, observouse que na análise entre os momentos pré-operatório e um mês pós-operatório, não houve alteração significativa; observamos uma média em valores de pontuação discretamente maior em nível de incapacidade da análise realizada um mês após a cirurgia em comparação à inicial, conforme demonstrado na Figura 2.

Após seis meses, houve queda estatisticamente significativa nos valores de incapacidade observados, porém não existe alteração significativa quando comparados momentos de observação de seis meses e um ano de pós-operatório (Tabela 2).

Não se observou relação estatisticamente significativa quando avaliados em conjunto o sexo e as escalas de dor e incapacidade; entretanto, foi identificada relação significativa em análises conjuntas no que diz respeito ao número de níveis artrodesados e avaliação de dor em VAS após seis meses do procedimento, assim como nas análises consecutivas. Na avaliação comparativa entre a quantidade de níveis artrodesados e os valores de incapacidade, observamos correlação estatisticamente significativa, na análise tardia posterior a dois anos de cirurgia. 


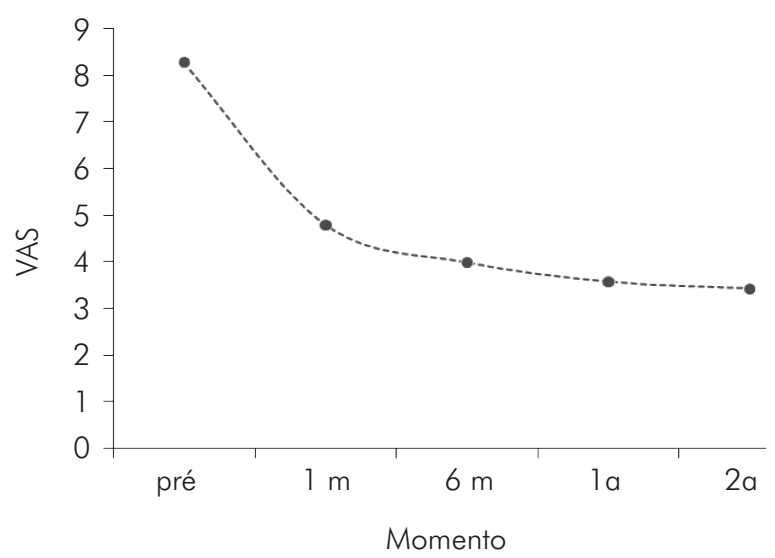

Figura 1

Avaliação prospectiva de dor (escala VAS).

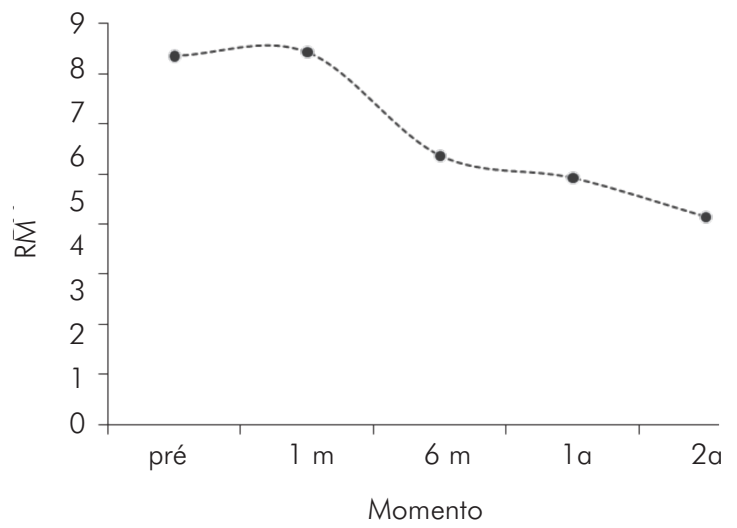

Figura 2

Avaliação prospectiva de incapacidade (Roland Morris).

\section{DISCUSSÃO}

A ECL é uma afecção crônica que acomete pacientes que possuem muitas vezes patologias associadas que contribuem para as limitações nas atividades da vida diária e provocam dor. Não foi objetivo deste estudo selecionar pacientes com quadro clínico, aspecto radiográfico, idade ou nível de comprometimento medular semelhantes, mas avaliar se o procedimento, quando bem indicado, gera impacto positivo sobre a incapacidade e queixas álgicas dos pacientes.

Pacientes com estenose de canal lombar, de acordo com a literatura, tornam-se sintomáticos e são submetidos à descompressão em torno da quinta ou sexta década de vida ${ }^{7}$. Em nossa análise, a idade média dos pacientes no momento da cirurgia foi de 66,55 anos. Muitas séries demonstram um predomínio do sexo masculino de aproximadamente $56 \%{ }^{12}$; este estudo encontrou um predomínio de $57,89 \%$ de mulheres e $42,10 \%$ de homens.

Observamos através da análise prospectiva das escalas VAS e Roland Morris uma melhora significativa nas comparações com valores observados em avaliações pré-operatórias, exceto nas avaliações pareadas de valores de Roland Morris pré-operatórios e um mês após o procedimento cirúrgico. É possível que esta constatação se deva à incapacidade temporária inerente ao período pós-operatório mais recente, no qual o paciente se recupera e inicia a reabilitação após a descompressão e artrodese lombar realizada, procedimento que agrega relativa morbidade nesta situação.

$\mathrm{Na}$ análise da dor por meio da escala VAS, observouse uma melhora significativa dos valores de pontuação de dor nas avaliações longitudinais em relação aos níveis pré-operatórios. Entretanto, as análises comparativas não apresentaram diferença estatisticamente significativa quando realizadas pareadas entre momentos de observação seis meses após o procedimento cirúrgico. Isto demonstra uma tendência à estabilização da melhora progressiva do quadro álgico durante observação de seis meses a dois anos.

Avaliamos em conjunto de pacientes com quantidades diversas em níveis de comprometimento vertebral, bem como gravidade variável de compressão. Entretanto, persiste na literatura a ausência de consenso sobre a relação estabelecida entre grau de estenose e agravamento dos sintomas ${ }^{3}$.

Não foi utilizado um grupo controle nesta avaliação; a evolução comparativa entre o tratamento conservador e cirúrgico é tema de discussão ainda sem definições bem estabelecidas. Segundo Kim et al., ${ }^{13}$ a história natural da ECL não é completamente conhecida devido à falta de estudos longitudinais prospectivos que documentam a evolução clínica de pacientes não-tratados. Entretanto, existem estudos que documentam de maneira criteriosa resultados em comparações a longo prazo, evidenciando possível superioridade do tratamento cirúrgico em relação ao tratamento não-cirúrgico ${ }^{14}$. Em concordância com os resultados da literatura, acreditamos que a indicação precisa tem fundamental importância nos resultados favoráveis do tratamento cirúrgico desta afecção $0^{2,8,15}$. Em análise envolvendo 30 pacientes submetidos à descompressão e artrodese por estenose de canal lombar, Padua et al., observaram, a longo prazo, a manutenção na melhora da qualidade de vida em avaliações oito anos após a cirurgia ${ }^{16}$ em pacientes criteriosamente diagnosticados.

$\mathrm{O}$ estudo Maine de coluna lombar ${ }^{14,17,18}$ acompanhou 81 pacientes tratados cirurgicamente por estenose de canal lombar em três momentos distintos; em análise após um ano do procedimento houve melhora em 77\% dos casos em relação ao período em vigência de tratamento conservador prévio; após quatro anos, 70\% dos pacientes referiam melhora clínica e, após oito anos, 54\% dos pacientes referiam manutenção dos benefícios clínicos obtidos após a intervenção cirúrgica. Amundsen et al. ${ }^{19}$ observaram melhora da queixa principal, avaliada como desfecho "positivo" em 89\% dos pacientes após um ano de tratamento cirúrgico; em $84 \%$ dos pacientes 
após quatro anos e em $71 \%$ dos pacientes após dez anos. Assim como outras análises demonstradas em literatura, observou-se o benefício inicial e uma tendência à estabilização do quadro com o decorrer desta observação, com acompanhamento de dois anos.

\section{CONCLUSÕES}

A cirurgia descompressiva com artrodese posterolateral com uso de parafusos pediculares na coluna lombar é eficiente na melhora de dor e incapacidade em avaliação dois anos após o procedimento.

\section{REFERÊNCIAS}

1. Sirvanci M, Bhatia M, Ganiyusufoglu KA, Duran C, Tezer M, Ozturk C, et al. Degenerative lumbar spinal stenosis: correlation with Oswestry Disability Index and MR Imaging. Eur Spine J. 2008;17(5):679-85.

2. Zouboulis P, Karageorgos A, Dimakopoulos P, Tyllianakis M, Matzaroglou C, Lambiris E. Functional outcome of surgical treatment for multilevel lumbar spinal stenosis. Acta Orthop. 2006;77(4):6706. Erratum in: Acta Orthop. 2007;78(6):862. Panagiotis, Zouboulis E [corrected to Zouboulis, Panagiotis]; Athanasios, Karageorgos [corrected to Karageorgos, Athanasios]; Panagiotis, Dimakopoulos [corrected to Dimakopoulos, Panagiotis]; Minos, Tyllianakis [corrected to Tyllianakis, Minos]; Charis, Matzaroglou [corrected to Matzaroglou, Charis]; Elias, Lambiris [corrected to Lambiris, Elias].

3. Arbit E, Pannullo S. Lumbar stenosis: a clinical review. Clin Orthop Relat Res. 2001;(384):137-43. Review.

4. Taylor VM, Deyo RA, Cherkin DC, Kreuter W. Low back pain hospitalization. Recent United States trends and regional variations. Spine (Phila Pa 1976). 1994;19(11):1207-12; discussion 13.

5. Fu YS, Zeng BF, Xu JG. Longterm outcomes of two different decompressive techniques for lumbar spinal stenosis. Spine (Phila Pa 1976). 2008;33(5):514-8.

6. Weiner DK. Office management of chronic pain in the elderly. Am J Med. 2007;120(4):306-15.

7. Atlas SJ, Delitto A. Spinal stenosis: surgical versus nonsurgical treatment. Clin Orthop Relat Res. 2006;443:198207.
8. Szpalski M, Gunzburg R. Lumbar spinal stenosis in the elderly: an overview. Eur Spine J. 2003;12 Suppl 2:S170-5.

9. Simotas AC. Nonoperative treatment for lumbar spinal stenosis. Clin Orthop Relat Res. 2001;(384):153-61.

10.Collins SL, Moore RA, McQuay HJ. The visual analogue pain intensity scale: what is moderate pain in millimetres? Pain. 1997;72(1-2):95-7.

11. Roland M, Morris R. A study of the natural history of low-back pain. Part II: development of guidelines for trials of treatment in primary care. Spine (Phila Pa 1976). 1983;8(2):145-50.

12.Katz JN, Dalgas M, Stucki G, Lipson SJ. Diagnosis of lumbar spinal stenosis. Rheum Dis Clin North Am. 1994;20(2):471-83. Review.

13. Kim JH, Lee HM, Kim HS, Moon ES, Park JO, Lee KJ, et al. Life expectancy after lumbar spine surgery: one-to eleven-year follow-up of 1015 patients. Spine (Phila $\mathrm{Pa}$ 1976). 2008;33(19):2116-21; discussion 2122-3.

14.Atlas SJ, Deyo RA, Keller RB, Chapin AM, Patrick DL, Long MJ, et al. The Maine Lumbar Spine Study, Part III. 1-year outcomes of surgical and nonsurgical management of lumbar spinal stenosis. Spine (Phila Pa 1976). 1996;21(15):1787-94; discussion 1794-5. Comment in: Spine (Phila Pa 1976). 1997;22(4):463-4. Spine (Phila Pa 1976). 1997;22(5):579-80.

15.Fritz JM, Delitto A, Welch WC, Erhard RE. Lumbar spinal stenosis: a review of current concepts in evaluation, management, and outcome measurements. Arch Phys Med Rehabil. 1998;79(6):700-8.
16.Padua L, Caliandro P, Padua R, Prezioso V, Aulisa GA, Mastontuoni $\mathrm{G}$, et al. Quality of life of patients operated on for lumbar stenosis: a long-term follow-up. Acta Neurochir (Wien). 2007;149(3):275-8; discussion 278-9.

17.Atlas SJ, Keller RB, Robson D, Deyo RA, Singer DE. Surgical and nonsurgical management of lumbar spinal stenosis: four-year outcomes from the maine lumbar spine study. Spine (Phila Pa 1976). 2000;25(5):556-62.

18.Atlas SJ, Keller RB, Wu YA, Deyo RA, Singer DE. Long-term outcomes of surgical and nonsurgical management of lumbar spinal stenosis: 8 to 10 year results from the maine lumbar spine study. Spine (Phila Pa 1976). 2005;30(8):936-43. Comment in: Spine (Phila Pa 1976). 2005;30(8):847-9.

19.Amundsen T, Weber H, Lilleas F, Nordal HJ, Abdelnoor M, Magnaes B. Lumbar spinal stenosis. Clinical and radiologic features. Spine (Phila $\mathrm{Pa}$ 1976). 1995;20(10):1178-86.

\begin{tabular}{l}
\hline Correspondência \\
Edgar Santiago Valesin Filho \\
Praça Ituzaingó, 120, apto. 151 \\
CEP: 03334-020 - São Paulo (SP), Brasil \\
Tel.: (1 1) 2671-3298 \\
E-mail: edgarsvf@hotmail.com
\end{tabular}

